\title{
Trial Disease Maximum Number of Actual Assessments
}

National Cancer Institute

\section{Source}

National Cancer Institute. Trial Disease Maximum Number of Actual Assessments. NCI

Thesaurus. Code C117706.

The upper limit in number of actual trial disease assessments during a planned period of time. 